\title{
Molecular evolution of the ent-kaurenoic acid oxidase gene in Oryzeae
}

\author{
Yanhua Yang ${ }^{1,2}$ and Keping Chen ${ }^{1}$ \\ ${ }^{1}$ Institute of Life Sciences, Jiangsu University, Zhenjiang, Jiangsu Province, China. \\ ${ }^{2}$ State Key Laboratory of Systematic and Evolutionary Botany, Institute of Botany, \\ Chinese Academy of Sciences, Beijing, China.
}

\begin{abstract}
We surveyed the substitution patterns in the ent-kaurenoic acid oxidase (KAO) gene in 11 species of Oryzeae with an outgroup in the Ehrhartoidaea. The synonymous and non-synonymous substitution rates showed a high positive correlation with each other, but were negatively correlated with codon usage bias and GC content at third codon positions. The substitution rate was heterogenous among lineages. Likelihood-ratio tests showed that the nonsynonymous/synonymous rate ratio changed significantly among lineages. Site-specific models provided no evidence for positive selection of particular amino acid sites in any codon of the KAO gene. This finding suggested that the significant rate heterogeneity among some lineages may have been caused by variability in the relaxation of the selective constraint among lineages or by neutral processes.
\end{abstract}

Key words: codon usage bias, ent-kaurenoic acid oxidase (KAO), positive selection, rate heterogeneity, substitution rate.

Received: July 21, 2011; Accepted: November 9, 2011.

\section{Introduction}

Gibberellins (GAs) are an important class of plant hormones involved in the regulation of various growth and developmental processes in higher plants (Appleford et al., 2006). The absence of GAs results in dwarfism in some plant species. ent-kaurenoic acid oxidase (KAO), a member of the CYP88A subfamily of cytochrome P450 enzymes, catalyzes a three-step reaction in the gibberellin biosynthetic pathway from ent-kaurenoic acid to GA12 (Helliwell et al., 2001). A primary goal of molecular evolutionary studies is to estimate the rate of DNA mutation and elucidate the mechanisms of molecular evolution. Such studies frequently involve a comparison of orthologous DNA fragments among species to determine evolutionary rates and an assessment of the evolutionary processes involved, e.g., natural selection, rate heterogeneity of lineages and mutational biases. Analysis of the molecular evolutionary patterns of different genes provides understanding of the evolutionary processes and pressures experienced by particular lineages.

The tribe Oryzeae (Poaceae) includes approximately 12 genera and more than 70 species distributed throughout tropical and temperate regions of the world (Clayton and Renvoize, 1986; Vaughan, 1994). In the genus Oryza, the Asian cultivated rice (Oryza sativa L.) is one of the world's most important crops and a primary food source for more

Send correspondence to Yanhua Yang. Institute of Life Sciences, Jiangsu University, 301 Xuefu Road, 212013 Zhenjiang, Jiangsu Province, P.R. China. E-mail: yanhuayang@126.com. than one-half of the world's population (Chandler and Wessler, 2001). This species has become a model monocotyledon in scientific research and its entire genome has been sequenced. Other members of the Oryzeae are also of economic importance, including wild species of Oryza that can be used in the genetic improvement of rice.

Analysis of the substitution patterns in the $K A O$ gene can provide insights into the driving forces that have led to evolutionary change in this gene in Oryzeae. In addition, the identification of patterns of molecular evolution in the $K A O$ gene can improve our understanding of the evolutionary history of some Oryzeae species. In this work, we examined the heterogeneity of the substitution rate in the $K A O$ gene among various genera and species of Oryzeae and sought to identify the possible causes of such heterogeneity. We also sought for evidence of natural selection in the exon regions of the $K A O$ gene.

\section{Materials and Methods}

\section{Plant material}

A portion of the $K A O$ gene was isolated and sequenced from members of the rice tribe (Oryzeae) (Table 1). Eleven diploid species were selected to represent the major phylogenetic lineages of Oryzeae (Figure S1, Supplementary Material) (Guo and Ge, 2005). These consisted of seven Oryza species representing six diploid genome types, namely, Oryza sativa (AA), O. meridionalis (AA), O. punctata (BB), O. officinalis (CC), O. australiensis (EE), O. brachyantha (FF), O. granulata (GG), and one 
Table 1 - Species used in this study.

\begin{tabular}{lccl}
\hline Species & Genome & Accession $^{\mathrm{a}}$ & Country \\
\hline Oryza sativa & A & japonica & GenBank \\
O. meridionalis & A & 105282 & Australia \\
O. punctata & B & 103903 & Tanzania \\
O. officinalis & C & 104972 & China \\
O. australiensis & E & 105263 & Australia-PNAS \\
O. brachyantha & F & 105151 & Sierra Leone-PNAS \\
O. granulata & G & M8-15 & Ledong, Hainan \\
Leersia tisserantti & - & 105610 & Cameroon \\
Chikusichloa aquatica & - & 106186 & Japan \\
Rhynchoryza subulata & - & 100913 & Argentina \\
Luziola leiocarpa & - & 82043 & Argentina \\
Ehrharta erecta & - & 218290 & South Africa \\
\hline
\end{tabular}

${ }^{a}$ All accessions were obtained from the International Rice Research Institute at Los Banos, Philippines.

species from each of four other genera in the tribe Oryzeae (Leersia tisserantti, Chikusichloa aquatica, Luziola leiocarpa, and Rhynchoryza subulata) (Table 1). Ehrharta erecta, a species in the tribe Ehrhartoideae, which is a sister tribe to the Oryzeae, was used as an outgroup (GPWG, 2001; Guo and Ge, 2005). Plastid, mitochondrial and nuclear gene sequences have been used to establish the phylogeny of the Oryzeae (Ge et al., 1999; Guo and Ge, 2005; Tang et al., 2010) and have provided an important framework for the study of molecular evolution in this group (Figure S1, Supplementary Material).

\section{DNA extraction, amplification and sequencing}

Total DNA was isolated from silica-gel dried or fresh leaves as described by Ge et al. (1999). A 1-2 kb fragment of the $K A O$ gene containing several exons and introns was obtained by using the polymerase chain reaction (PCR) in conjunction with the forward primer KAOF (5'-CAGGA CGTTCATGTTCAGCAG-3') and the reverse primers KAOR1 (5'-TCGTCGCCAAGCAGTTGTC-3') and KAOR2 (5'-GCCAAGCAGTTGTCCAC-3') (Figure 1). The PCR was done in a total volume of $25 \mu \mathrm{L}$ that contained 5-50 ng of template DNA, $0.2 \mu \mathrm{M}$ of each primer, $200 \mu \mathrm{M}$ of each dNTP, $10 \mathrm{mM}$ Tris- $\mathrm{HCl}$ (pH 8.3), $50 \mathrm{mM}$ $\mathrm{KCl}, 1.5 \mathrm{mM} \mathrm{MgCl}_{2}$ and $0.75 \mathrm{U}$ of ExTaq DNA polymer-

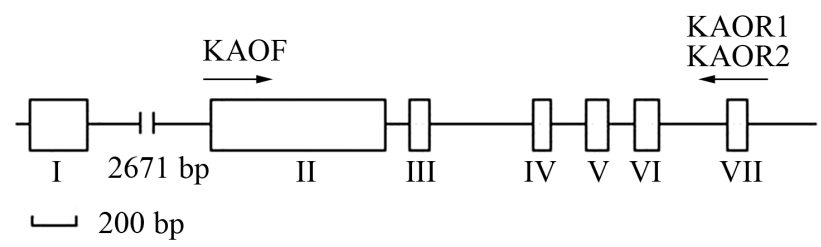

Figure 1 - Schematic diagram of the $K A O$ gene and the regions sequenced in this study. Boxes and lines indicate exons and introns, respectively. Exon numbers are labeled with the roman numbers. Locations of primers are shown above the diagram. ase (TaKaRa, Shiga, Japan). Amplifications were done in a $\mathrm{T}$ gradient $96 \mathrm{U}$ thermocycler (Biometra, Göttingen, Germany) as follows: 3 min at $94{ }^{\circ} \mathrm{C}$, followed by 33 cycles of $30 \mathrm{~s}$ at $94^{\circ} \mathrm{C}, 30 \mathrm{~s}$ at $56^{\circ} \mathrm{C}, 2.5 \mathrm{~min}$ at $72{ }^{\circ} \mathrm{C}$ and a final extension at $72{ }^{\circ} \mathrm{C}$ for $10 \mathrm{~min}$. Further internal primers used for sequencing were: KAO707F 5'-ACCGTCTTCCTCC AGGAGAAC-3' $\quad\left(\mathrm{Tm}=61.9{ }^{\circ} \mathrm{C}\right), \quad \mathrm{KAO} 931 \mathrm{~F}$ 5'-GATGCACTTCCTCTCACAG-3' $\left(\mathrm{Tm}=57.6^{\circ} \mathrm{C}\right)$ and KAO1478F 5'-CGTCAACATCTCCTTCGTGTC-3' $\left(\mathrm{Tm}=60^{\circ} \mathrm{C}\right)$ (Yang et al., 2009). All of the sequences were deposited in GenBank under accession numbers EF577665-EF577670 and EU179429-EU179435 (Table 2).

\section{Sequence analysis}

Sequences were aligned using ClustalX v.1.81 (Thompson et al., 1997) and refined by manual adjustment based on the predicted amino acid sequence. The amino acid sequences (excluding introns) were sufficiently conserved across the 12 species to provide unambiguous alignments. We examined the possibility of sequence saturation using DAMBE v.4.5.45 (Xia and Xie, 2001). Pairwise synonymous and non-synonymous substitutions per site $\left(d_{\mathrm{S}}\right.$ and $d_{\mathrm{N}}$ ) among the 11 species were estimated for the coding regions of the $K A O$ gene.

The extent of codon usage bias often reflects the degree of selective constraint in a gene (Sharp, 1991; Sharp et al., 1986). To measure the extent of codon usage bias, we estimated the effective number of codons (ENC) and codon bias index (CBI) using DnaSP v.4.10.9 (Rozas and Rozas, 1999). The ENC values range from 20 (only one codon is used for each amino acid, i.e., the codon bias is maximal) to 61 (all synonymous codons for each amino acid are equally used, i.e., there is no codon bias) (Wright, 1990). The CBI values range from 0 (uniform use of synonymous codons) to 1 (maximum codon bias) (Morton, 1993). Variation in the rate of synonymous substitution among genes may be related to codon use (Sharp, 1991). Therefore, several parameters related to codon usage bias, such as the GC content at the first and second codon positions $(\mathrm{GC1}, 2)$, as well as third codon positions (GC3), were also estimated using DnaSP v.4.10.9 (Rozas and Rozas, 1999).

\section{Detecting rate heterogeneity among lineages}

The relative-rate test based on the method of Muse and Gaut (1994), as implemented in Hyphy (Pond et al., 2005), was used to detect variation in the synonymous and non-synonymous substitution rates along different lineages, with Ehrharta erecta as the reference sequence. This method examines substitution rates between two lineages with reference to a third outgroup lineage. In the first model, the two related taxa from the most recent common ancestor are constrained to have the same substitution rate. In the second model, the two lineages may have different substitution rates. A likelihood ratio test is used to test 


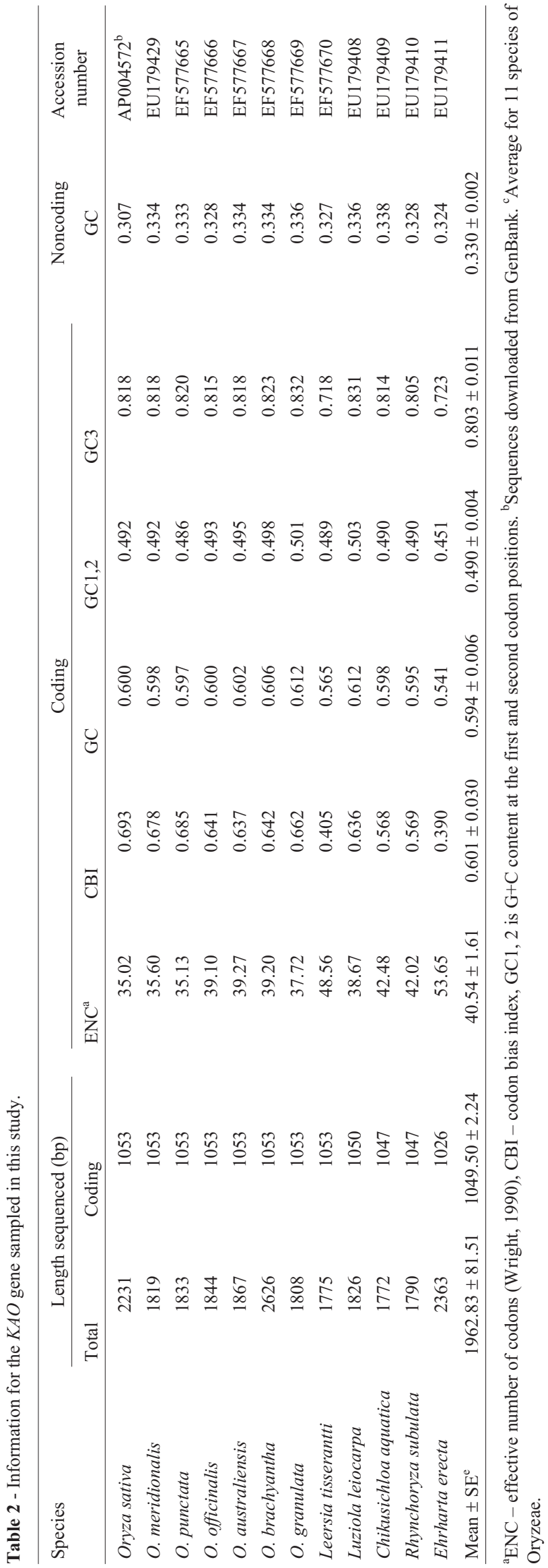

which of the models best explains the data (Muse and Gaut, 1994).

\section{Detection of positive selection}

The ratio $\omega\left(d_{\mathrm{N}} / d_{\mathrm{S}}\right)$ provides an effective means of detecting selection or selective pressure on a gene or gene region, with $\omega<1,=1$ and $>1$ indicating negative selection, neutral evolution and positive selection, respectively (Yang, 2006). We ran likelihood-based analyses using the CODEML program of PAML 4 (Yang, 2007) to explore the selective processes acting on the $K A O$ gene. First, we used the branch models to examine whether the evolutionary rates differed among lineages within the gene tree. The one ratio model (M0) assumes a single $\omega$ for all branches and all sites. However, the free ratio model (Mf) postulates an independent $\omega$ ratio for each branch of the tree. A likelihood ratio test (LRT) was used to decide whether there was a significant difference between M0 and Mf. The model with the higher likelihood value was assumed to be the better model (Bielawski and Yang, 2003; Yang and Nielsen, 1998).

We next used site-specific models to detect whether particular amino acid residues were subject to positive selection (Yang, 2006). The neutral model (M1a) classifies all of the sites into two categories, i.e., strict constraint $(0<\omega<1)$ (purifying selection) and neutral $(\omega=1)$. Based on M1a, the positive selection model (M2a) assumes a third category under positive selection $(\omega>1)$. The beta model (M7) assumes a beta distribution for the $\omega$ ratios over sites, and the beta and $\omega$ model (M8) increases the independent ratio estimated by the data. M8 and M2a assume positive selection and are compared with M7 and M1a, respectively. If the LRT is significant and there is a site with $\omega>1$ then positive selection is invoked for the gene (Bielawski and Yang, 2003; Yang, 2006).

\section{Results and Discussion}

Previous studies showed that the $K A O$ gene was a single-copy gene (Helliwell et al., 2001; Sakamoto et al., 2004; Yamaguchi, 2008) and the loss-of-function mutant exhibits a typical phenotype, indicating the functional importance of this enzyme in GA biosynthesis (Sakamoto et al., 2004). In view of the importance of comparing orthologous rather than paralogous genes when estimating substitution rates, we initially examined this issue and found that the $K A O$ gene was orthologous in all of the species analyzed. The similarity of the aligned coding regions ranged from $87.5 \%$ to $99.5 \%$ (Figure S2, Supplementary Material). Sequences of the $K A O$ gene were isolated from all of the Oryzeae species and from the outgroup, Ehrharta erecta. The sequenced regions ranged in size from $1772 \mathrm{bp}$ to $2626 \mathrm{bp}$ and their aligned coding regions varied from $1047 \mathrm{bp}$ to $1053 \mathrm{bp}$ (Table 2). The total GC content and the GC content of the third position of the codons (GC3) were 
similar across species. Table 2 summarizes the sequence data for this gene.

\section{Codon usage bias and its correlation with GC3 and substitution rates}

Codon usage bias has been important in studies of molecular evolution because it provides examples of weak selection at the molecular level. CBI and ENC were calculated to measure the degree of codon usage bias. CBI showed a marked negative correlation with ENC $\left(\mathrm{r}^{2}=0.958, \mathrm{p}<0.0001\right)($ Figure 2A) such that both CBI and ENC could be used to measure the degree of codon usage bias. In this study, ENC was used to measure the degree of codon usage bias.

To determine the relative effects of mutation pressure versus natural selection on codon composition, we examined the relationship between the GC content at third codon positions (GC3) and the $\mathrm{GC}$ content at the first and second codon positions $(\mathrm{GC} 1,2)$. The $\mathrm{GC}$ content of $\mathrm{GC} 1,2$ ranged from $48.9 \%$ to $50.3 \%$, which there was a tendency of positive correlation with GC3 $\left(\mathrm{r}^{2}=0.227\right)$ but this was not significant $(\mathrm{p}=0.139)$ (Figure $2 \mathrm{~F})$. This pattern of base composition suggests that the $\mathrm{GC}$ content is most likely the result of mutation pressure since natural selection acts differently on different codon positions (Shackelton et al., 2006). Interestingly, after excluding L. tisserantti, GC1,2 showed a significant positive correlation with GC3 $\left(r^{2}=0.604, p<0.05\right)$ (data not shown), which further confirmed that these changes were most likely the result of mutation pressure. $d_{\mathrm{S}}$ was positively correlated with $d_{\mathrm{N}}$ $\left(r^{2}=0.498, p<0.05\right)($ Figure 2D), as also observed in other organisms (Bielawski et al., 2000; Dunn et al., 2001; Hurst and Williams, 2000; Kusumi et al., 2002), and negatively correlated with codon bias $\left(\mathrm{r}^{2}=0.713, \mathrm{p}<0.05\right)$ (Figure $2 \mathrm{~B})$ and $\mathrm{GC} 3\left(\mathrm{r}^{2}=0.796, \mathrm{p}<0.001\right)$ (Figure 2E). The negative correlation between $d_{\mathrm{S}}$ and codon usage bias may be explained by natural selection (Bielawski et al., 2000; Smith and Eyre-Walker, 2001; Urrutia and Hurst, 2001) since codon usage bias is a primary factor in $d_{\mathrm{S}}$ variation among genes and is thought to be under natural selection, perhaps because of the need to maintain accuracy or speed in translation (Yang and Gaut, 2011). There was also a tendency for $d_{\mathrm{N}}$ being negatively correlated with codon usage bias $\left(r^{2}=0.348\right)$ but this was not significant $(p=0.056)$ (Figure 2C). The latter would be consistent with sites that are functionally constrained and consequently conserved at the amino acid level. Such sites are also likely to experience stronger selection for translation accuracy and hence have a higher codon bias (Akashi, 2003). This might explain the negative correlation between $d_{\mathrm{N}}$ and codon bias observed here (though not significant), and by others in enteric bacteria (Rocha, 2004; Sharp, 1991), Drosophila (Betancourt and Presgraves, 2002), yeast (Drummond et al., 2005), and viruses (Duffy et al., 2008). The fact that $d_{\mathrm{N}}$ is correlated to codon bias suggests that codon bias might be used as a mea-
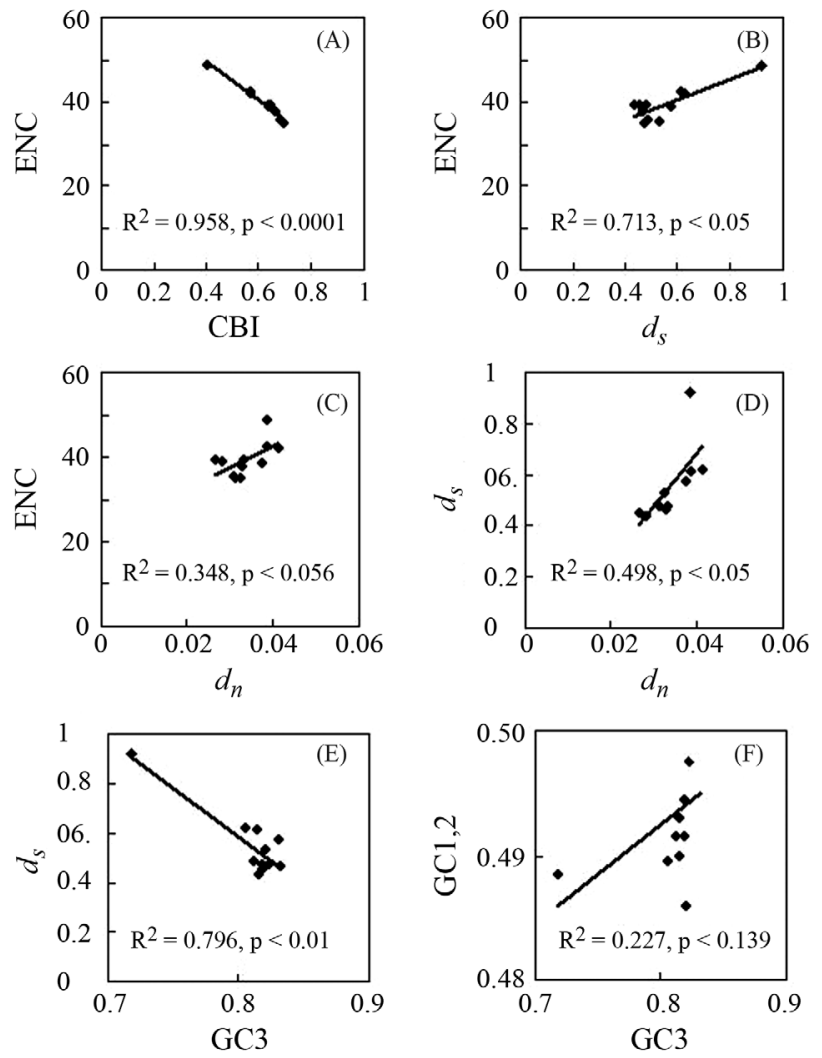

Figure 2 - The relationships between effective number of codons (ENC) and codon bias index (CBI) $(\mathrm{A})$, synonymous substitution rates $\left(d_{\mathrm{S}}\right)(\mathrm{B})$, and non-synonymous substitution rates $\left(d_{\mathrm{N}}\right)(\mathrm{C})$, between $d_{\mathrm{S}}$ and $d_{\mathrm{N}}(\mathrm{D})$ and third codon positions (GC3) (E), and between the first and second codon positions $(\mathrm{GC} 1,2)$ and $\mathrm{GC} 3(\mathrm{~F})$.

sure of the level of constraint upon a site or gene (Plotkin et al., 2004, 2006; Stoletzki and Eyre-Walker, 2007).

\section{The driving forces governing evolution of the $K A O$ gene in Oryzeae}

A codon-based approach showed that the free ratio model (Mf) had significantly higher likelihood scores $(\ln 4103.38)$ than the one ratio model (M0) $(\ln 4124.44)$ $(\mathrm{p}<0.001)$ (Table 3$)$. Although the $d_{\mathrm{N}} / d_{\mathrm{S}}$ ratios varied across lineages from 0.0001 to 0.358 (with one of the 21 lineages showing no predicted synonymous substitutions, i.e., the $d_{\mathrm{N}} / d_{\mathrm{S}}$ ratio was equal to 999.000 ), the estimated $d_{\mathrm{N}} / d_{\mathrm{S}}$ ratio for each lineage was less than 1 . The $\omega$ values were estimated to be 0.079 under the M0 model, suggesting that purifying selection or selection constraint best explained the molecular evolution of the $K A O$ gene, in agreement with the studies on anthocyanin pathway genes $(\mathrm{Lu}$ and Rausher, 2003; Rausher et al., 2008).

The branch model test is a very conservative test of positive selection because it averages the ratio across all sites. We therefore used site-specific codon models to examine whether there was positive selection on codon sites. The M2a and M8 models, which assume positive selection, were not significantly better than the null models M1a and 
Table 3 - Log likelihood values, $\omega$ ratios and parameter estimates for the $K A O$ gene in models with variable $\omega$ ratios among codon sites.

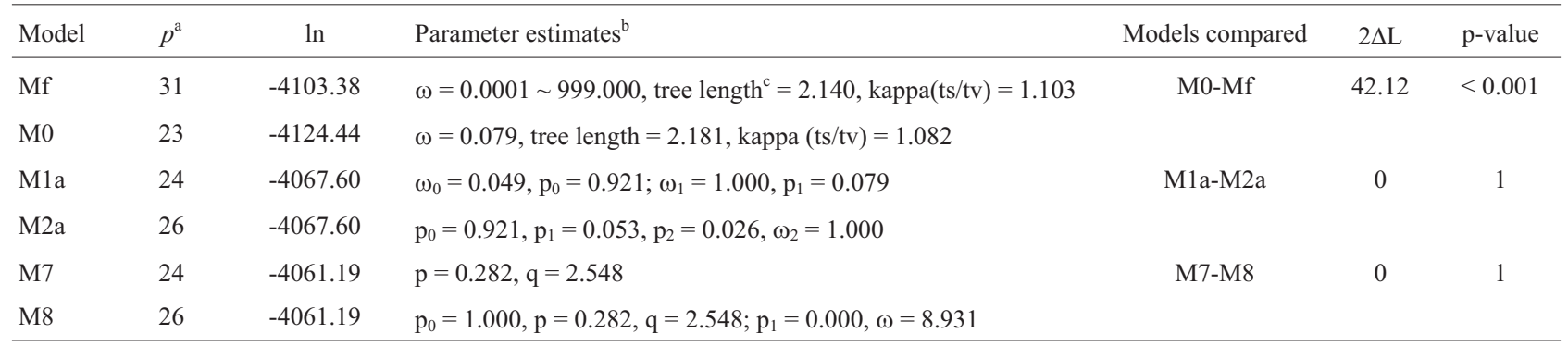

${ }^{\mathrm{a}} \mathrm{p}$ - number of parameters, $\ln$ - log-likelihood values of the data in each model. ${ }^{\mathrm{b}}$ Parameter estimates in different models.

${ }^{\mathrm{c}}$ Tree length is the sum of branch lengths.

$\mathrm{M} 7$ (for M1a vs. M2a, $2 \Delta \mathrm{L}=0, \mathrm{p}=1.0$; for $\mathrm{M} 7$ vs. M8, $2 \Delta=0, \mathrm{p}=1.0)$ (Table 3$)$. These results indicate that the $K A O$ gene is under strong selective constraint, thus ruling out the possibility of past episodes of positive selection on this gene. Previous studies have shown that variation in the evolutionary rate among nucleotide sites may be attributed to differences in the frequency of positive selection (Yang et al., 2000; Gaut et al., 2011) or in the magnitude of selective constraints (Li, 1997; Rausher et al., 1999, 2008).

In this study, the branch and codon models failed to detect any sign of positive selection for any lineage and codon of the $K A O$ gene, suggesting that the significant heterogeneity of some lineages was attributable mainly to the relaxed constraint among lineages or neutral processes rather than positive selection. However, the power to detect positive selection using the methods mentioned above may be low, especially when adaptive substitutions are spread across many amino acid sites (Pond et al., 2005; Rausher et al., 2008). Further investigations with alternative tests on intraspecific changes (Olsen et al., 2002; Whitt et al., 2002;
Flowers et al., 2007; Rausher et al., 2008) would be necessary to detect evidence of positive selection.

\section{Rate variation among lineages}

There was significant heterogeneity in the synonymous and non-synonymous substitution rates of the $K A O$ gene among lineages of the rice tribe (Table 4), especially in C. aquatica and L. leiocarpa. Among 55 relative-rate tests for synonymous substitutions, 11 comparisons were significant at the $5 \%$ or $1 \%$ level. At the same time, among 55 relative-rate tests for non-synonymous substitutions, the null hypothesis of rate homogeneity was rejected for 18 comparisons. In C. aquatica and L. leiocarpa $d_{\mathrm{N}}$ appeared to be decelerated, and $\operatorname{did} d_{\mathrm{S}}$ in $C$. aquatica. The significant slowdown in the rate of synonymous and non-synonymous substitutions in C. aquatica and L. leiocarpa lineages may reflect differences in the intensity of selection, i.e., the $K A O$ gene may be under different functional constraints in different lineages.

Several mechanisms could explain the observed rate heterogeneity, including life history traits such as genera-

Table 4 - Results of 110 relative-rate tests for $d_{\mathrm{S}}$ (lower triangle) and $d_{\mathrm{N}}$ (upper triangle). Rejection of rate equality is indicated by $*$ at the 0.05 level, $* *$ at the 0.01 level, or *** at the 0.001 level. Ehrharta erecta was used as the outgroup in all comparisons. Species names that were inferred to have evolved more quickly in each pairwise comparison are indicated in the table by the first letter of the genus name and the first three letters of the species name.

\begin{tabular}{|c|c|c|c|c|c|c|c|c|c|c|c|}
\hline & Osat & Omer & Opun & Ooff & Oaus & Obra & Ogra & Ltis & Llei & Caqu & Rsub \\
\hline Osat & - & & & & & & & & $* * *$ Osat & $* *$ Osat & \\
\hline Omer & & - & & & & *Omer & $*$ Ogra & & $* * *$ Omer & $* * *$ Omer & \\
\hline Opun & & & - & & & *Opun & & & $* * *$ Opun & **Opun & \\
\hline Ooff & & & & - & & & & & $* *$ Ooff & $* *$ Ooff & \\
\hline Oaus & & & & & - & & & & $* *$ Oaus & *Oaus & \\
\hline Obra & & & & & & - & & & $*$ Obra & & \\
\hline Ogra & & & & & & & - & & *Ogra & & \\
\hline Ltis & & & & & & & & - & $* *$ Ltis & ${ }^{*}$ Ltis & \\
\hline Llei & & & & & & & & & - & & \\
\hline Caqu & $* * *$ Osat & ***Omer & $* * *$ Opun & $* *$ Ooff & $* *$ Oaus & $* *$ Obra & $* * *$ Ogra & $* * *$ Ltis & $* *$ Llei & - & * Rsub \\
\hline Rsub & & & & & & & $*$ Ogra & & & *Rsub & - \\
\hline
\end{tabular}

Caqu - Chikusichloa aquatica, Llei - Luziola leiocarpa, Ltis - Leersia tisserantti, Oaus - O. australiensis, Obra - O. brachyantha, Ogra - O. granulata, Omer-O. meridionalis, Ooff - O. officinalis, Opun - O. punctata, Osat - O. sativa and Rsub - Rhynchoryza subulata. 
tion time, biochemical features such as efficiency of DNA repair machinery, and environmental variables such as energy and temperature (Eyre-Walker and Gaut, 1997; Li, 1997; Brown et al., 2005; Soria-Hernanz et al., 2008). Rate heterogeneity may also result from differences in population size since variation in population size can alter evolutionary rates within a lineage (Eyre-Walker and Gaut, 1997; Lynch and Conery, 2003) and vice versa. Variation in the nucleotide substitution rates of the $K A O$ gene significantly changed the $\omega$ ratios of the respective lineages. These features of the $K A O$ gene in Oryzeae resulted from the influence of various factors that affected the evolution of these species and their ancestors. A detailed knowledge of these factors will help us to understand the evolutionary history of Oryzeae species.

\section{Conclusions}

The results of this study showed that codon usage bias was negatively correlated with synonymous and nonsynonymous substitution rates, a finding consistent with the importance of codon usage. CBI was positively correlated with ENC, thus confirming the similarity of $\mathrm{CBI}$ and ENC as parameters for measuring the degree of codon usage bias. There was considerable heterogeneity in the nucleotide substitution rates of the $K A O$ gene and this significantly affected the $\omega$ ratios of the respective lineages. There was no positive selection and no positively selected codons in this gene, a finding indicative of substantial selective constraint. These features of nucleotide substitutions in the $K A O$ gene reflected the influence of various factors on the evolution of many Oryzeae species and their ancestors.

\section{Acknowledgments}

YYH thanks his instructor, Prof. Song Ge, for help with the design and execution of this work. We thank Fu-Min Zhang, Hong-Zheng Sun and other members of Dr. Ge's group for their help during the experimental work and data analysis. We thank the International Rice Research Institute (Los Banos, Philippines) for providing the seed samples. This work was supported by the National Basic Research Program of China (2007CB815704), the Ministry of Agriculture Transgenic Major Project (2009ZX08012018B) and the Scientific Research Promotion Fund for the Talents of Jiangsu University (11JDG049).

\section{References}

Akashi H (2003) Translational selection and yeast proteome evolution. Genetics 164:1291-1303.

Appleford N, Evans D, Lenton J, Gaskin P, Croker S, Devos K, Phillips A and Hedden P (2006) Function and transcript analysis of gibberellin-biosynthetic enzymes in wheat. Planta $223: 568-582$.
Betancourt AJ and Presgraves DC (2002) Linkage limits the power of natural selection in Drosophila. Proc Natl Acad Sci USA 99:13616-13620.

Bielawski JP and Yang Z (2003) Maximum likelihood methods for detecting adaptive evolution after gene duplication. $\mathrm{J}$ Struct Funct Genomics 3:201-212.

Bielawski JP, Dunn KA and Yang Z (2000) Rates of nucleotide substitution and mammalian nuclear gene evolution. Approximate and maximum-likelihood methods lead to different conclusions. Genetics 156:1299-1308.

Brown JM, Pauly GB and May G (2005) Increased rates of molecular evolution in an equatorial plant clade: An effect of environment or phylogenetic nonindependence? Evolution 59:238-242.

Chandler VL and Wessler S (2001) Grasses. A collective model genetic system. Plant Physiol 25:1155-1156.

Clayton WD and Renvoize SA (1986) Genera Graminum. Kew Bulletin Additional Series XIII:1-389.

Drummond DA, Bloom JD, Adami C, Wilke CO and Arnold FH (2005) Why highly expressed proteins evolve slowly. Proc Natl Acad Sci USA 102:14338-14343.

Duffy S, Shackelton LA and Holmes EC (2008) Rates of evolutionary change in viruses: Patterns and determinants. Nat Rev Genet 9:267-276.

Dunn KA, Bielawski JP and Yang Z (2001) Substitution rates in Drosophila nuclear genes: Implications for translational selection. Genetics 157:295-305.

Eyre-Walker A and Gaut BS (1997) Correlated rates of synonymous site evolution across plant genomes. Mol Biol Evol 14:455-460.

Flowers JM, Sezgin E, Kumagai S, Duvernell DD, Matzkin LM, Schmidt PS and Eanes WF (2007) Adaptive evolution of metabolic pathways in Drosophila. Mol Biol Evol 24:13471354.

Gaut B, Yang L, Takuno S and Eguiarte LE (2011) The patterns and causes of variation in plant nucleotide substitution rates. Annu Rev Ecol Evol Syst 42:245-266.

Ge S, Sang T, Lu BR and Hong DY (1999) Phylogeny of rice genomes with emphasis on origins of allotetraploid species. Proc Natl Acad Sci USA 96:14400-14405.

GPWG (2001) Phylogeny and subfamilial classification of the grasses (Poaceae). Ann MO Bot Gard 88:373-457.

Guo YL and Ge S (2005) Molecular phylogeny of Oryzeae (Poaceae) based on DNA sequences from chloroplast, mitochondrial, and nuclear genomes. Am J Bot 92:1548-1558.

Helliwell CA, Chandler PM, Poole A, Dennis ES and Peacock WJ (2001) The CYP88A cytochrome P450, ent-kaurenoic acid oxidase, catalyzes three steps of the gibberellin biosynthesis pathway. Proc Natl Acad Sci USA 98:2065-2070.

Hurst LD and Williams EJ (2000) Covariation of GC content and the silent site substitution rate in rodents: Implications for methodology and for the evolution of isochores. Gene 261:107-114.

Kusumi J, Tsumura Y, Yoshimaru H and Tachida H (2002) Molecular evolution of nuclear genes in Cupressacea, a group of conifer trees. Mol Biol Evol 19:736-747.

Li WH (1997) Molecular Evolution. Sinauer Associates, Sunderland, $487 \mathrm{pp}$.

Lu Y and Rausher MD (2003) Evolutionary rate variation in anthocyanin pathway genes. Mol Biol Evol 20:1844-1853. 
Lynch M and Conery JS (2003) The origins of genome complexity. Science 302:1401-1404.

Morton BR (1993) Chloroplast DNA codon use: Evidence for selection at the $p s b A$ locus based on tRNA availability. J Mol Evol 37:273-280.

Muse SV and Gaut BS (1994) A likelihood approach for comparing synonymous and nonsynonymous nucleotide substitution rates, with application to the chloroplast genome. Mol Biol Evol 11:715-724.

Olsen KM, Womack A, Garrett AR, Suddith JI and Purugganan MD (2002) Contrasting evolutionary forces in the Arabidopsis thaliana floral developmental pathway. Genetics 160:1641-1650.

Plotkin JB, Dushoff J and Fraser HB (2004) Detecting selection using a single genome sequence of $M$. tuberculosis and $P$. falciparum. Nature 428:942-945.

Plotkin JB, Dushoff J, Desai MM and Fraser HB (2006) Codon usage and selection on proteins. J Mol Evol 63:635-653.

Pond SL, Frost SD and Muse SV (2005) HyPhy: Hypothesis testing using phylogenies. Bioinformatics 21:676-679.

Rausher M, Lu Y and Meyer K (2008) Variation in constraint versus positive selection as an explanation for evolutionary rate variation among anthocyanin genes. J Mol Evol 67:137-144.

Rausher MD, Miller RE and Tiffin P (1999) Patterns of evolutionary rate variation among genes of the anthocyanin biosynthetic pathway. Mol Biol Evol 16:266-274.

Rocha EPC (2004) Codon usage bias from tRNA's point of view: Redundancy, specialization, and efficient decoding for translation optimization. Genome Res 14:2279-2286.

Rozas J and Rozas R (1999) DnaSP ver. 3: An integrated program for molecular population genetics and molecular evolution analysis. Bioinformatics 15:174-175.

Sakamoto T, Miura K, Itoh H, Tatsumi T, Ueguchi-Tanaka M, Ishiyama K, Kobayashi M, Agrawal GK, Takeda S, Abe K, et al. (2004) An overview of gibberellin metabolism enzyme genes and their related mutants in rice. Plant Physiol 134:1642-1653.

Shackelton LA, Parrish CR and Holmes EC (2006) Evolutionary basis of codon usage and nucleotide composition bias in vertebrate DNA viruses. J Mol Evol 62:551-563.

Sharp PM (1991) Determinants of DNA sequence divergence between Escherichia coli and Salmonella typhimurium: Codon usage, map position, and concerted evolution. J Mol Evol 33:23-33

Sharp PM, Tuohy TM and Mosurski KR (1986) Codon usage in yeast: Cluster analysis clearly differentiates highly and lowly expressed genes. Nucleic Acids Res 14:5125-5143.

Smith NG and Eyre-Walker A (2001) Synonymous codon bias is not caused by mutation bias in $\mathrm{G}+\mathrm{C}$-rich genes in humans. Mol Biol Evol 18:982-986.

Soria-Hernanz DF, Braverman JM and Hamilton MB (2008) Parallel rate heterogeneity in chloroplast and mitochondrial genomes of Brazil nut trees (Lecythidaceae) is consistent with lineage effects. Mol Biol Evol 25:1282-1296.

Stoletzki N and Eyre-Walker A (2007) Synonymous codon usage in Escherichia coli: Selection for translational accuracy. Mol Biol Evol 24:374-381.
Tang L, Zou XH, Achoundong G, Potgieter C, Second G, Zhang DY and Ge S (2010) Phylogeny and biogeography of the rice tribe (Oryzeae): Evidence from combined analysis of 20 chloroplast fragments. Mol Phylogenet Evol 54:266-277.

Thompson JD, Gibson TJ, Plewniak F, Jeanmougin F and Higgins DG (1997) The CLUSTAL_X windows interface: Flexible strategies for multiple sequence alignment aided by quality analysis tools. Nucleic Acids Res 25:4876-4882.

Urrutia AO and Hurst LD (2001) Codon usage bias covaries with expression breadth and the rate of synonymous evolution in humans, but this is not evidence for selection. Genetics 159:1191-1199.

Vaughan DA (1994) The Wild Relative of Rice: A Genetic Resources Handbook. International Rice Research Institute, Manila, $137 \mathrm{pp}$.

Whitt SR, Wilson LM, Tenaillon MI, Gaut BS and Buckler ES (2002) Genetic diversity and selection in the maize starch pathway. Proc Natl Acad Sci USA 99:12959-12962.

Wright F (1990) The "effective number of codons" used in a gene. Gene 87:23-29.

Xia X and Xie Z (2001) DAMBE: Software package for data analysis in molecular biology and evolution. J Hered 92:371373.

Yamaguchi S (2008) Gibberellin metabolism and its regulation. Annu Rev Plant Biol 59:225-251.

Yang L and Gaut BS (2011) Factors that contribute to variation in evolutionary rate among Arabidopsis genes. Mol Biol Evol 28:2359-2369.

Yang YH, Zhang FM and Ge S (2009) Evolutionary rate patterns of the gibberellin pathway genes. BMC Evol Biol 9:206.

Yang Z (2006) Computational Molecular Evolution. Oxford University Press, Oxford, $357 \mathrm{pp}$.

Yang Z (2007) PAML 4: Phylogenetic analysis by maximum likelihood. Mol Biol Evol 24:1586-1591.

Yang Z and Bielawski JP (2000) Statistical methods for detecting molecular adaptation. Trends Ecol Evol 15:496-503.

Yang Z and Nielsen R (1998) Synonymous and nonsynonymous rate variation in nuclear genes of mammals. J Mol Evol 46:409-418

\section{Supplementary Material}

The following online material is available for this article:

Figure S1 - Phylogeny of the rice tribe (Oryzeae) obtained from the combined $A d h 2$ and GPA1 sequences by Bayesian inference using the $\mathrm{TrN}+\mathrm{G}$ model (Guo and Ge, 2005).

Figure S2 - Alignment of coding sequences of the $K A O$ gene in 12 species.

This material is available as part of the online article from http:/www.scielo.br/gmb.

Associate Editor: Adriana S. Hemerly

License information: This is an open-access article distributed under the terms of the Creative Commons Attribution License, which permits unrestricted use, distribution, and reproduction in any medium, provided the original work is properly cited. 


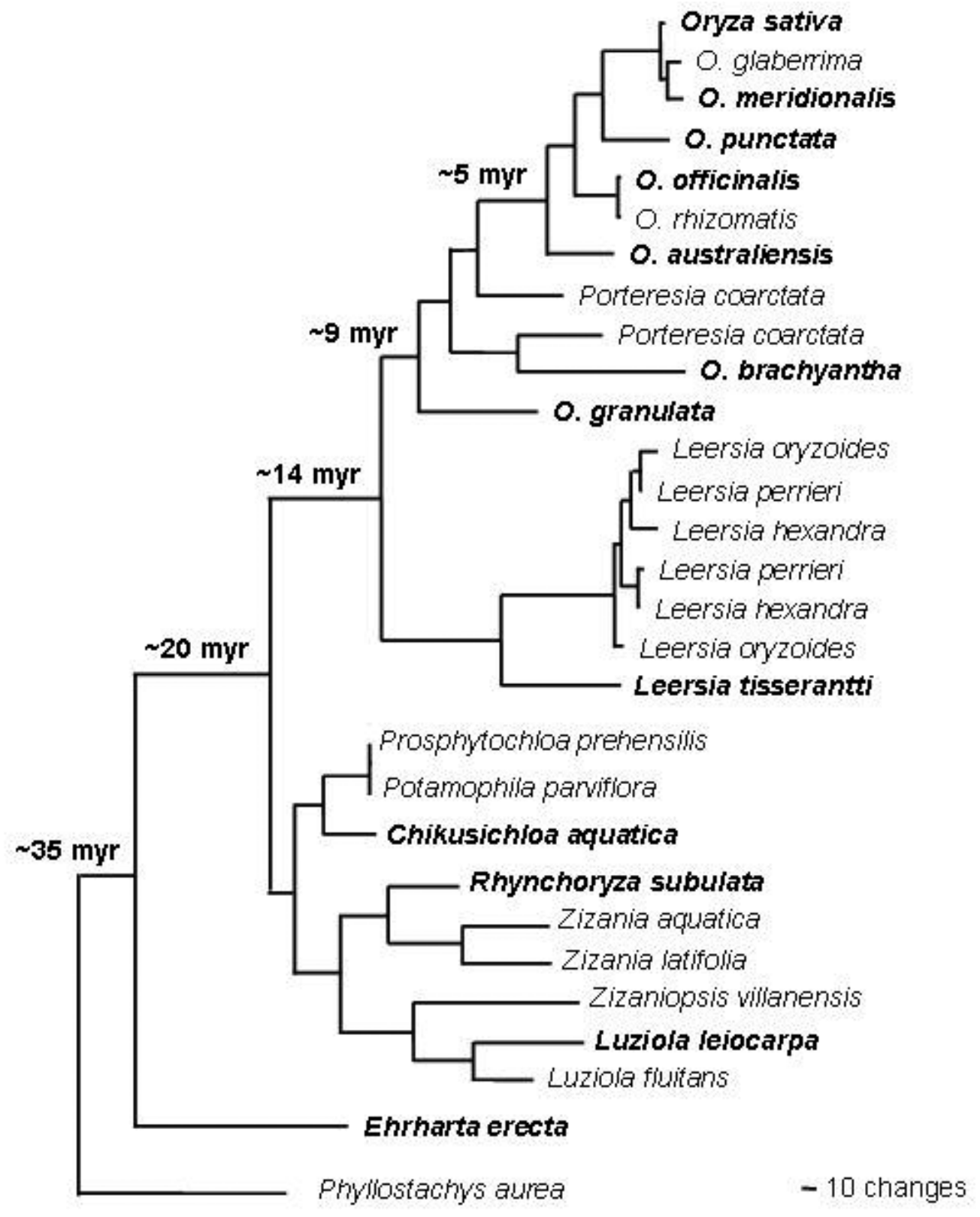




\section{O.sativa_kao}

$\begin{array}{llllll}10 & 20 & 30 & 40 & 50 & 60\end{array}$

GGCTGGCCCAAGGCCACCGTCACCCTCATCGGCCCCAAATCCTTCGTCAACATGTCCT AC

O.meridionalis_kao

GGCTGGCCCAAGGCCACCGTCACCCTCATCGGCCCCAAATCCTTCGTCAACATGTCCT AC

O.punctata_kao

GGCTGGCCCAAGGCCACCGTCACCCTCATCGGCCCCAAATCCTTCGTCAACATGTCCT

AC

O.officinalis_kao

GGCTGGCCCAAGGCCACCGTCACCCTCATCGGCCCCAAATCCTTCGTCAACATGTCCT

AC

O.australiensis_kao

GGCTGGCCCAAGGCCACCGTCACCCTCATCGGCCCCAAATCCTTCGTCAACATGCCCT AC

O.brachyantha_kao

GGCTGGCCCAAGGCGACCATCACTCTCATCGGCCCCAAGTCCTTCGTCAACATGCCCT

AC

O.granulata_kao

GGATGGCCCAAGGCCACCGTCACCCTCATCGGCCCCAAGTCCTTCGTCAGCATGCCCT AC

L.tisseranttikao

GGATGGCCGAAAGCGACGGTGACACTGATCGGGCCAAAATCGTTCGTGAGCATGCCGT

AC

C.aquatica_kao

GGCTGGCCCAAGGCAACGGTCACGCTCATCGGCCGCAAGTCGTTCGTGAGCATGCCG TAC

R.subulata_kao

GGCTGGCCCAAGGCCACCGTCAATCTCATCGGCCGCAAGTCGTTCGTGAGCATGCCAT AC

L.leiocarpa_kao

GGCTGGCCCAAGGCCACCGTCACACTCATCGGCCCCAAGTCCTTCGTCAGCATGCCGC AC

E.erecta_kao

GGCTGGCCCAAGTCGACGGTGACGCTCCTCGGCAACAACTCATTCATAAGCTTGCCCC AC

Clustal Consensus

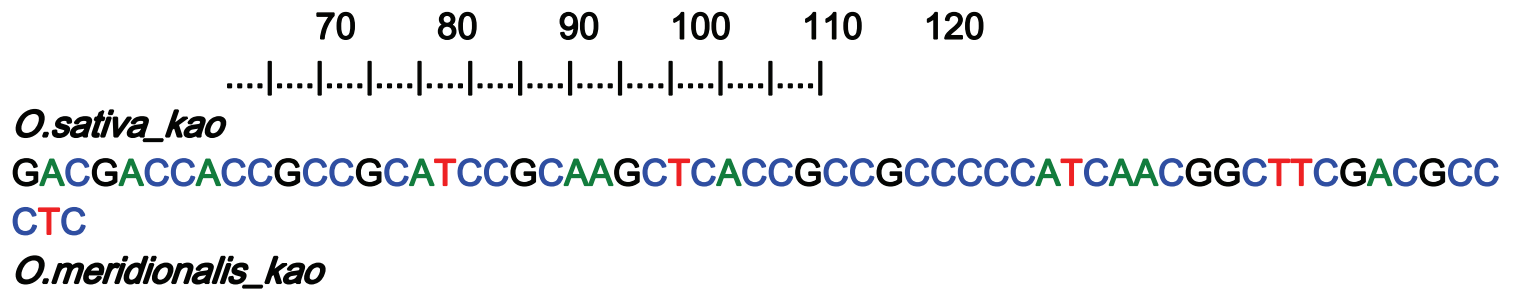


GACGACCACCGCCGCATCCGCAAGCTCACCGCCGCCCCCATCAACGGCTTCGACGCC CTC

O.punctata_kao

GACGACCACCGCCGCATCCGCAAGCTCACCGCCGCCCCCATCAACGGCTTCGACGCC CTC

O.officinalis_kao

GACGACCACCGCCGCATCCGCAAGCTCACCGCCGCCCCCATCAACGGCTTCGACGCG

CTC

O.australiensis_kao

GACGACCACCGCCGCATCCGCAAGCTCACGGCGGCGCCCATCAACGGCTTCGACGCC CTC

O.brachyantha_kao

GACGACCACCGCCGCCTGCGCAAGCTCACGGCCGCGCCCATCAACGGCTTCGACGCG CTC

O.granulata_kao

GACGACCACCGCCGCCTCCGCAAGCTCACCGCCGCGCCCATCAACGGCTTCGACGCG CTC

L.tisseranttikao

GACGATCACCGGCGAATCCGCAAGCTGACGGCGGCGCCGATCAACGGCTTCGACGCA

CTC

C.aquatica_kao

GAGGAACACCGGCGCTTGCGCAAGCTCACGGCGGCGCCCATCAACGGCTTCGAGGCG CTC

R.subulata_kao

GAGGATCACCGGCGGCTGCGCAAGCTCACGGCGGCTCCCATCAACGGCTTCGAGGCG CTC

\section{L.leiocarpa_kao}

GACGACCACCGCCGGCTCCGCAAGCTCACGGCGGCGCCCATCAACGGCTTCGACGCG CTC

E.erecta_kao

GACGACCACCGGCGTCTGCGCAAGCTGACCGCGGCGCCAATCAACGGCTTTGATTCAC TG

Clustal Consensus

O.sativa_kao

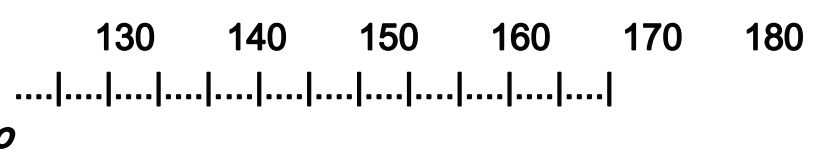

ACCACCTACCTCTCCTTCATCGACCAGACCGTCGTCGCCTCCCTCCGCCGCTGGTCCT CG

O.meridionalis_kao

ACCACCTACCTCTCCTTCATCGACCAGACCGTCGTCGCCTCCCTCCGCCGCTGGTCCT CG

O.punctata_kao

ACCACCTACCTCTCCTTCATCGACCACACCGTCGTCTCCACGCTCCGCCGCTGGTCCT

CC

O.officinalis_kao 
AACACCTACCTCGCCTTCATCGACCAAACCGTCGTCGCCACTCTCCGCCGCTGGTCCT CG

O.australiensis_kao

ACCACCTACCTCGCCTTCATCGACCAGACCGTCGTCGCTACGCTCCGCCGCTGGTCGT CG

\section{O.brachyantha_kao}

ACCACCTACCTCGGCTTCATCGACCGCACCGTCGTCGACACGCTCCGCCGCTGGTCGT CG

O.granulata_kao

ACCACCTACCTCGGCTTCATCGACCACACCGTCGTCGCCTCGCTCCGCCGCTGGTCGG AG

L.tisseranttikao

ACAACCTACCTCTCCTTCATCGACCAAACCGTCGTCGCCACGCTCCGCCGCTGGTCGG AA

C.aquatica_kao

ACCACCTACCTGGGCTTCATCGACCAGACCGTTGTGTCCACGCTGCGCCGCTGGTCGG AG

R.subulata_kao

ACCACCTACCTGGGCTTCATCGACCAGACCGTCGTGGCCACGCTGCGCCGCTGGTCG GAG

L.leiocarpa_kao

ACCACCTACCTCGCCTTCATCGACCAGACCGTCGTCTCCACGCTCCGCCGCTGGTCCG AC

E.erecta_kao

ACCACGTACCTCGGATTCATCGACAAGACCGTCGTGTCGACGCTGAGTCGGTGGTCGG AC

Clustal Consensus

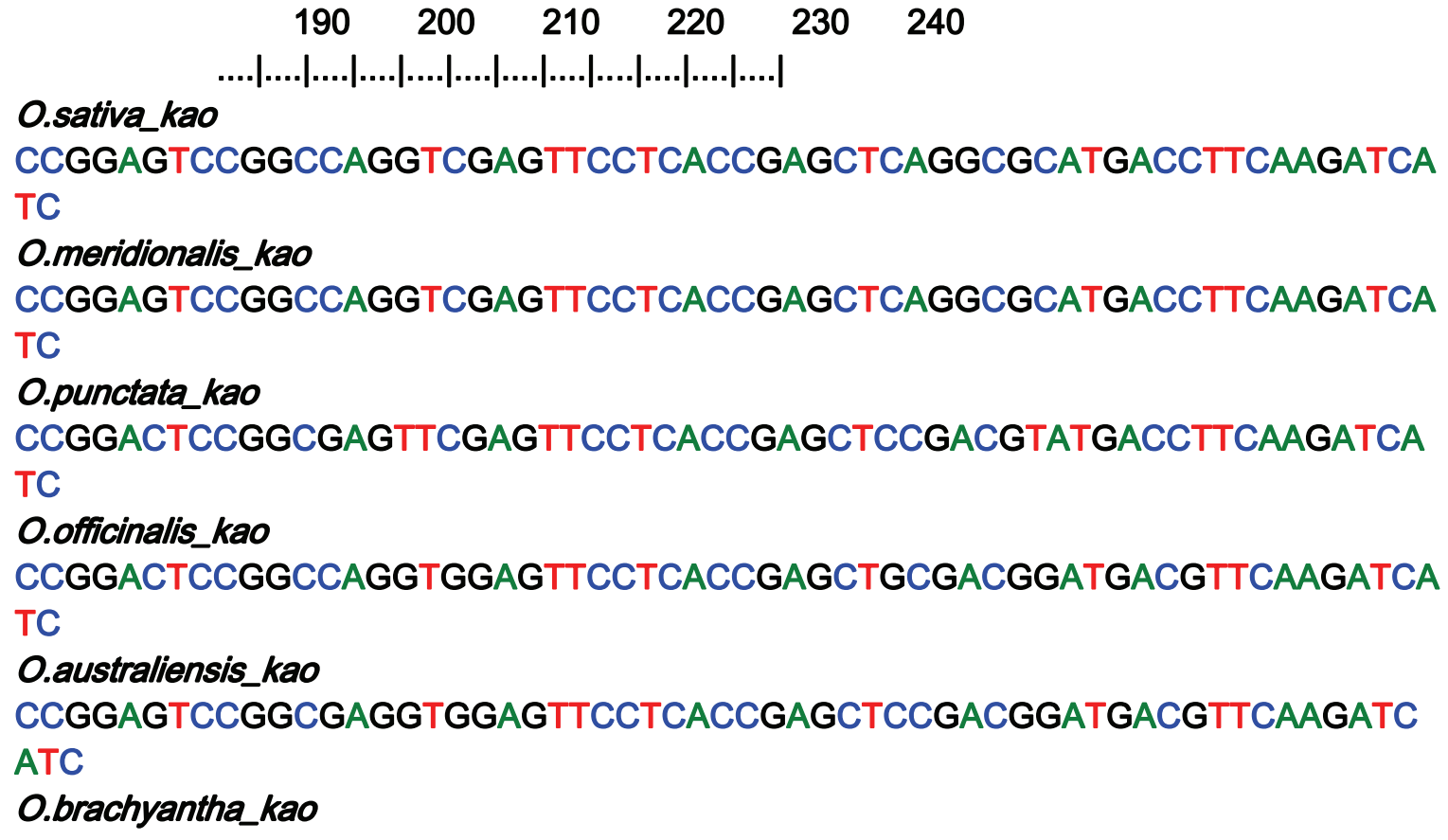


GAGCCAGCCGCCGAGGTCGAGTTCCTCACCGAGCTGCGCCGGATGACCTTCAAGATC ATC

O.granulata_kao

CCCGGCGCAGGCGAGGTCGAGTTCCTCACCGAGCTCCGCCGCATGACGTTCAAGATC ATC

L.tisserantti_kao TCC---

TCCGGCGAGATCAAATTCCTCACCGAGCTCCGCCGCATGACGTTCAAGATCATC

C.aquatica_kao

GCCGGCGAGGTGGAGTTCCTCACGGAGCTGCGGCGGATGACCTTCAAGATCATT

R.subulata_kao

GCCGGCGAGGTGGAGTTCCTCACCGAGCTGCGGCGGATGACGTTCAAGATCATC

L.leiocarpa_kao

GGCGCCGGCGAGGTCGAGTTCCTCACCGAGCTCCGCCGCATGACCTTCAAGATCATC

E.erecta_kao

GGGGGGAAGGAGATGGAGTTGATCCTCACGGAGATGCGGCGGATGAACTTCAAGGTC ATC

Clustal Consensus

$\begin{array}{llllll}250 & 260 & 270 & 280 & 290 & 300\end{array}$

O.sativa_kao

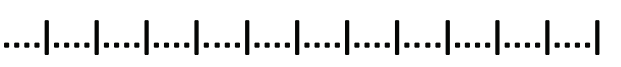

GTCCAGATCTTCATGAGCGGCGCCGACGACGCCACCATGGAGGCCCTGGAGCGGAGC

TAC

O.meridionalis_kao

GTCCAGATCTTCATGAGCGGCGCCGACGACGCCACCATGGAGGCCCTGGAGCGGAGC TAC

O.punctata_kao

GTCCAGATCTTCATGAGCGGCGCCGACGACGCCACCATGGAGGCCCTCGAGCGGAGC TAC

O.officinalis_kao

GTCCAGATCTTCATGAGCGGCGCCGACGACGCCACCATGGAGGCCCTGGAGCGGAGC TAC

O.australiensis_kao

GTCCAGATCTTCATGAGCGGCGCCGACGATGCCACCATGGAGGCGCTGGAGCGGAGC TAC

O.brachyantha_kao

GTCCAGATCTTCATGAGCGGCGCCGACGACCGCACCATGGAGGCCCTCGAGCGGAGC TAC

O.granulata_kao

GTCCAGATCTTCATGAGCGGCGCCGACGACCGCACCATGGAGGCGCTGGAGCGGAGC TAC

L.tisseranttikao

GTCCAGATCTTCATGAGCGGCGCCGACGATCGAACAATGGAGGCATTGGAGAGGAGCT $\mathrm{AC}$

C.aquatica_kao

GTCCAGATCTTCATGAGCGGCGCCGACGACCGCACCATGGAGGCGCTGGAGAGGAGC 
TAC

R.subulata_kao

GTCCAGATCTTCATGAGCGGCGCCGACGACCGTACCATGGAGGCGCTGGAGAGGAGC

TAC

L.leiocarpa_kao

GTCCAGATCTTCATGAGCGGCGCCGACGACCGCACCATGGAGGCGCTGGAGCGGAGC TAC

E.erecta_kao

GTCGAGATCTTCATGGGCGGCGCCGACGACAAAATCATGGATGACCTAGAAGGAAGCT AC

Clustal Consensus

O.sativa_kao

$\begin{array}{llllll}310 & 320 & 330 & 340 & 350 & 360\end{array}$

ACCGACCTCAACTACGGCATGCGCGCCATGGCCATCAACCTCCCCGGCTTCGCCTACT

$\mathrm{AC}$

O.meridionalis_kao

ACCGACCTCAACTACGGCATGCGCGCCATGGCCATCAACCTCCCCGGCTTCGCCTACT

$\mathrm{AC}$

O.punctata_kao

ACCGACCTCAACTACGGCATGCGCGCCATGGCCATCAACCTCCCGGGCTTCGCCTACC AC

O.officinalis_kao

ACCGACCTCAACTACGGGATGCGCGCCATGGCCATCAACCTCCCAGGGTTCGCCTACC

$\mathrm{AC}$

O.australiensis_kao

ACCGACCTCAACTACGGGATGCGCGCCATGGCCATCAACCTCCCCGGGTTCGCCTACC $\mathrm{AC}$

O.brachyantha_kao

ACCGACCTCAACTACGGCATGCGCGCCATGGCCATCAACCTCCCCGGCTTCGCCTACC $\mathrm{AC}$

O.granulata_kao

ACCGACCTCAACTACGGCATGCGCGCCATGGCCATCAACCTCCCGGGCTTCGCCTACC $\mathrm{AC}$

L.tisseranttikao

ACCGATCTCAACTATGGGATGCGAGCCATGGCGATCAACATCCCCGGATTCGCCTACC

AT

C.aquatica_kao

ACCGACCTCAACTACGGCATGCGCGCCATGGCCATCAACCTCCCCGGCTTCGCCTACC $\mathrm{AC}$

R.subulata_kao

ACAGACCTCAACTACGGCATGCGCGCCATGGCCATCAACCTCCCCGGCTTCGCCTACC AC

L.leiocarpa_kao

ACCGACCTCAACTATGGCATGCGCGCCATGGCCATCAACCTCCCCGGCTTCGCCTACC 
AC

E.erecta_kao

ACCGAGCTCAACTATGGCCTGCGCGCCATGGCTATCAACCTCCCCGGGTTCGCCTACC AC

Clustal Consensus

$\quad 370 \quad 380 \quad 390 \quad 400 \quad 410420$
O.sativa_kao
CGCGCGCTCAGGGCTCGCCGGAAGCTCGTGTCCGTGCTGCAGGGTGTGCTCGACGGC
CGG
O.meridionalis_kao
CGCGCGCTCAGGGCTCGCCGGAAGCTCGTGTCCGTGCTGCAGGGTGTGCTCGACGGT
CGG
O.punctata_kao
CGCGCCCTCAGGGCTCGCCGGAAGCTCGTCTCCGTCCTGCAGGGGGTGCTCCACGCC AGG

O.officinalis_kao

CGCGCGCTCAGGGCTCGCCGGAAGCTGGTGTCCGTGCTGCAGGGGGTGCTCGACGG CAGG

O.australiensis_kao

CGCGCGCTCAGAGCTCGCCGGAAGCTGGTGTCCGTGCTGCAGGGGGTGCTCGACGG

CAGG

O.brachyantha_kao

AGGGCGCTCCGGGCTCGCCGGAAGCTGGTGTCCGTGCTGCAGGGCGTGCTCGACGG CAGG

O.granulata_kao

AGGGCGCTCAGGGCTCGCCGGCGGCTGGTGTCCGTGCTGCAGGGCGTGCTCGACAG CAGG

L.tisseranttikao

CGCGCTCTCAGGGCTCGCCGGAAGCTCGTCGCCGTTCTGCAGGGAGTTCTCGACGGC AGG

C.aquatica_kao

AGGGCCCTCAAGGCTCGCCGGAAGCTGGTGTCCGTGCTGCAGGGCGTGCTGGACAGC AGG

R.subulata_kao

AGGGCCCTCAAGGCTCGCCGGAAGCTGGTGTCCGTGCTGCAGGGTGTGCTGGACAGC AGG

L.leiocarpa_kao

CGGGCGCTCAGGGCTCGCCGGAGGCTGGTCGCCGTGCTGCAGGCGGTGCTCAACGG CCGG

E.erecta_kao

CGAGCTCTGAAATCTCGCAAGAAGCTAGTGTCTGCCATGCAGGCAATGCTGGACGGGA GG

Clustal Consensus 


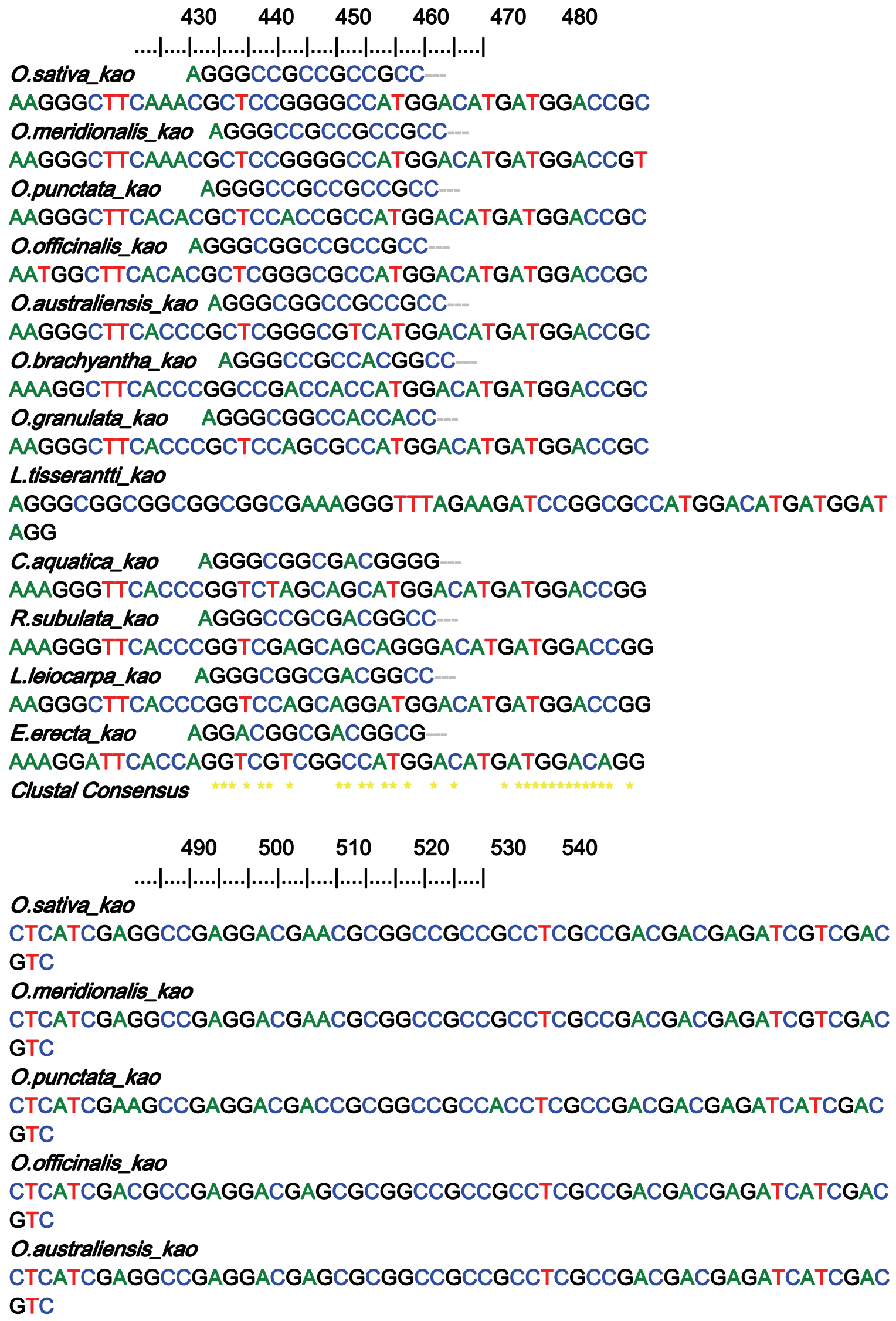




\section{O.brachyantha_kao}

CTCATCGAGGCCCAGGACGAGCGCGGCCGCCGGCTCGCCGACGACGAGATCATCGAC GTC

O.granulata_kao

CTCATCGAGGCCGAGGACGACCGCGGCCGCCGCCTCGCCGACGACGAGATCATCGAC GTC

\section{L.tisseranttikao}

CTTATCGAGGCTGAGGATGAGCGTGGACGGCGGCTCGCCGACGACGAGATCATCGAT GTG

C.aquatica_kao

CTGATCGAGGCGGAGGACGAGCGCGGCCGGCGGCTGGCCGACGACGAGATCATCGA CGTC

R.subulata_kao

CTGATCGAGGCCGAGGACGAGCGCGGCCGGCGGCTCGGCGACGACGAGATCATCGA CGTC

L.leiocarpa_kao

CTGATCGAGGCGGAGGACGAGCGCGGCCGCCGCCTCGCCGACGACGAGATCATCGA CGTC

\section{E.erecta_kao}

TTGATCGAGGTGGAGGATGAGCATGGGCGGCGGCTTAGAGACGATGAGATCATCGACA TC

Clustal Consensus

O.sativa_kao

$\begin{array}{llllll}550 & 560 & 570 & 580 & 590 & 600\end{array}$

CTCATCATGTACCTCAACGCCGGCCACGAGTCCTCCGGCCACATCACCATGTGGGCCA $\mathrm{CC}$

O.meridionalis_kao

CTCATCATGTACCTCAACGCCGGCCACGAGTCCTCCGGCCACATCACCATGTGGGCCA CC

O.punctata_kao

CTCATCATGTACCTCAACGCCGGCCACGAGTCCTCCGGCCACATCACCATGTGGGCCA CC

O.officinalis_kao

CTCATCATGTACCTCAACGCCGGCCACGAGTCCTCCGGCCACATCACCATGTGGGCCA CC

O.australiensis_kao

CTCATCATGTACCTCAACGCCGGCCACGAGTCCTCCGGCCACATCACCATGTGGGCCA $\mathrm{CC}$

O.brachyantha_kao

CTCATCATGTACCTCAACGCCGGCCACGAGTCCTCCGGCCACATCACCATGTGGGCCA CC

O.granulata_kao

CTCATCATGTACCTCAACGCCGGCCACGAGTCCTCCGGCCACATCACCATGTGGGCCA CC 


\section{L.tisseranttikkao}

CTCATCATGTACCTCAACGCCGGCCATGAATCTTCCGGCCATATCACCATGTGGGCCAC C

C.aquatica_kao

CTCATCATGTACCTCAACGCCGGCCACGAGTCCTCCGGCCACATCACCATGTGGGCCA $\mathrm{CC}$

R.subulata_kao

CTCGTCATGTACCTCAACGCCGGCCATGAGTCCTCCGGCCACATCACCATGTGGGCCA $\mathrm{CC}$

L.leiocarpa_kao

CTCATCATGTACCTCAACGCCGGCCACGAGTCCTCCGGCCACATCACCATGTGGGCCA CC

E.erecta_kao

CTCAACATGTACATCAACGCCGGCCACGAGTCCTCCATACACATCACCATGTGGGCTAC $\mathrm{T}$

Clustal Consensus

O.sativa_kao

$\begin{array}{llllll}610 & 620 & 630 & 640 & 650 & 660\end{array}$

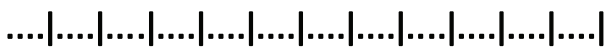

GTCTTCCTCCAGGAGAACCCCGACATCTTCGCAAGAGCAAAGGCTGAGCAAGAGGAGA TC

O.meridionalis_kao

GTCTTCCTCCAGGAGAACCCCGACATCTTCGCAAGAGCAAAGGCTGAGCAAGAGGAGA TC

O.punctata_kao

GTCTTCCTCCAAGAGAACCCCGACATCTTCGCAAGAGCAAAGGCGGAGCAAGAGGAGA TC

O.officinalis_kao

GTCTTCCTCCAGGAGAACCCCGACATCTTCGCAAGAGCAAAGGCCGAACAAGAGGAGA TC

O.australiensis_kao

GTCTTCCTCCAGGAGAACCCCGACATCTTCGCAACAGCAAAAGCGGAGCAAGAGGAGA TC

O.brachyantha_kao

GTCTTCCTCCAGGAGAACCCCGACATCTTCGCAAGGGCAAAGGCTGAGCAAGAGGAGA TC

O.granulata_kao

GTCTTCCTCCAGGAGAACCCCGACATCTTCGCAAGGGCCAAGGCGGAGCAAGAGGAG ATC

\section{L.tisseranttikao}

GTCTTCCTCCAGGAGAATCCCGACATCTTAGCAAGGGCAAAGGCTGAGCAAGAGGAGA TC

C.aquatica_kao

GTCTTCCTGCAGGAGAACCCCGAAATCTTTGCAAGGGCAAAGGCCGAGCAAGAGGAGA TC 


\section{R.subulata_kao}

GTCTTCCTCCAGGAGAACCCCGAAATCTTCGCAAGGGCAAAGGCCGAGCAAGAGGAAA $\mathrm{TC}$

\section{L.leiocarpa_kao}

GTCTTCCTGCAGGAGAACCCCGACATCTTCGCGAGGGCAAAGGCCGAGCAAGAAGAG ATC

\section{E.erecta_kao}

TTTTTCCTGCAAGAGAACCCGGACGTATTAGCAAGGGCAAAGGCCGGGCAAGAGGAGA TC

Clustal Consensus

O.sativa_kao

$$
\begin{aligned}
& \begin{array}{llllll}
670 & 680 & 690 & 700 & 710 & 720
\end{array}
\end{aligned}
$$

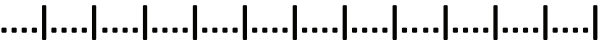

ATGAGAAGCATTCCAGCAACGCAGAACGGATTAACCCTCAGGGACTTCAAGAAGATGC

AC

O.meridionalis_kao

ATGAGAAGCATTCCAGCAACGCAGAACGGATTAACCCTCAGGGACTTCAAGAAGATGC

AC

O.punctata_kao

ATGAGAAGCATACCAGCAACGCAGAAGGGATTAACGCTCAGGGACTTCAAGAAGATGC

AT

\section{O.officinalis_kao}

ATGAGAAGCATACCAGCAACGCAGAAGGGATTAACGCTCAGGGACTTCAAGAAGATGC AC

\section{O.australiensis_kao}

ATGAGAAGCATACCAGCAACGCAGAAGGGATTAACCCTCAGGGACTTCAAGAAGATGC GC

O.brachyantha_kao

ATGAGAAGCATACCCGCAACGCAGAAGGGACTGACACTCAGGGACTTCAAGAAGATGC AG

O.granulata_kao

ATGAGAAGCATACCACCAACGCAGAAGGGACTCAACCTCAGGGACTTCAAGAAGATGC AG

\section{L.tisseranttikao}

ATGAGAAGCATACCACCAACGCAGAAGGGACTTACCCTTAGGGACTTCAAGAAGATGC AC

C.aquatica_kao

ATGAGAAGCATACCACCAACACAGAAGGGACTGAACCTCAGGGACTTCAAGAAGATGC AG

R.subulata_kao

ATGAGAAACATACCATCAACACAGAAGGGACTGAACCTCAGGGACTTCAAGAAGATGCA $\mathrm{T}$

L.leiocarpa_kao

ATGAGAAGCATACCACCAACACAGAAGGGGCTGAGCCTCAGGGACTTCAAGAAGATGC AG 
E.erecta_kao ATGAGAAGC CTGAGGGATTTCAGGAAGATGGAG Clustal Consensus

O.sativa_kao

$\begin{array}{cccccc}730 & 740 & 750 & 760 & 770 & 780 \\ \ldots . \ldots|\ldots| \ldots \mid & \end{array}$

TTCCTCTCACAGGTTGTCGACGAGACACTTCGCTGCGTCAACATCTCCTTCGTGTCCTT $\mathrm{C}$

O.meridionalis_kao

TTCCTCTCACAGGTTGTCGACGAGACACTTCGCTGCGTCAACATCTCCTTCGTGTCCTT $\mathrm{C}$ O.punctata_kao TTCCTCTCACAGGTTGTCGACGAGACACTTCGCTGCGTCAACATCTCCTTCGTGTCCTT $\mathrm{C}$ O.officinalis_kao TTCCTCTCACAGGTTGTCGACGAGACACTTCGCTGCGTCAACATCTCCTTCGTGTCCTT $\mathrm{C}$

O.australiensis_kao

TTCCTCTCACAGGTTATCGACGAGACACTTCGCTGCGTCAACATCTCCTTCGTGTCCTT $\mathrm{C}$

O.brachyantha_kao

TTCCTCTCCCAGGTTATCGATGAGACACTTCGCTGCGTCAACATCTCGTTCGTGTCATT $\mathrm{C}$ O.granulata_kao TTCCTCTCGCAGGTTATCGACGAGACGCTTCGCTGCGTCAACATCTCGTTTGTGTCCTT C

\section{L.tisseranttikao}

TTCCTCCAGCAGGTTGTCGACGAGACACTTCGCTGCGTCAACATCTCCTTCGTGTCCTT $\mathrm{C}$

C.aquatica_kao

TACCTCTCACAGGTTATCGACGAGACACTTCGCTGCGTCAACATCTCCTTTGTGTCCTT $\mathrm{C}$

R.subulata_kao TACCTCTCACAGGTTGTCGACGAGACACTTCGCTGCGTCAACATCTCGTTTGTGTCCTT C

L.leiocarpa_kao

TACCTCTCACAGGTTATCGACGAGACGCTTCGCTTTGTCAACATCTCGTTTGTGTCCTTC E.erecta_kao TACCTCTCACATGTTATCGACGAGACACTTCGCTTCGTCAACATGTCCTTTTTGTCGTTC Clustal Consensus

O.sativa_kao $\begin{array}{llllll}790 & 800 & 810 & 820 & 830 & 840\end{array}$

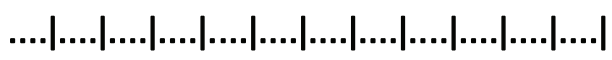
CGTCAGGCCACAAGAGACATCTTTGTGAACGGTTATCTTATCCCCAAGGGGTGGAAGG TT 


\section{O.meridionalis_kao}

CGTCAGGCCACAAGAGACATCTATGTGAACGGTTATCTGATCCCCAAGGGGTGGAAGG

TT

O.punctata_kao

CGTCAGGCCACAAGAGACATCTATGTGAACGGCTATCTGATCCCCAAGGGGTGGAAGG

TC

O.officinalis_kao

CGTCAGGCCACAAGAGACATCTATGTGAACGGCTATCTGATCCCCAAGGGGTGGAAGG

TT

O.australiensis_kao

CGCCAGGCCACAAGAGACATCTATGTGAACGGCTATCTGATACCCAAGGGCTGGAAGG

TT

O.brachyantha_kao

CGTCAGGCGACCAGAGACGTCTATGTGAACGGCTATCTGATACCCAAGGGCTGGAAGG

TT

O.granulata_kao

CGTCGGGCGACAAGAGACGTCTATGTGAACGGTTATCTGATACCCAAGGGTTGGAAGG

TT

L.tisseranttikkao

CGTCAGGCGACAAAAGACGTCTATGTGAACGGCTATCTGATACCCAAGGGCTGGAAGG

TT

C.aquatica_kao

CGCCAAGCGACACGAGACGTCTTTGTGAACGGCTATCTGATACCAAAGGGCTGGAAGG

TT

R.subulata_kao

CGTCAGGCGACCCGAGACGCCTTCGTGAACGGCTATCTGATACCAAAGGGCTGGAAG

GTT

L.leiocarpa_kao

CGTCAGGCAACACGCGACGTCTATGTGAACGGTTATCTGATACCAAAGGGCTGGAAGG

TT

E.erecta_kao

CGCCAGGCAACAAAAGACGTCTTTGTGAATGGCTATCTGATACCAAAGGGTTGGAAGGT

G

Clustal Consensus

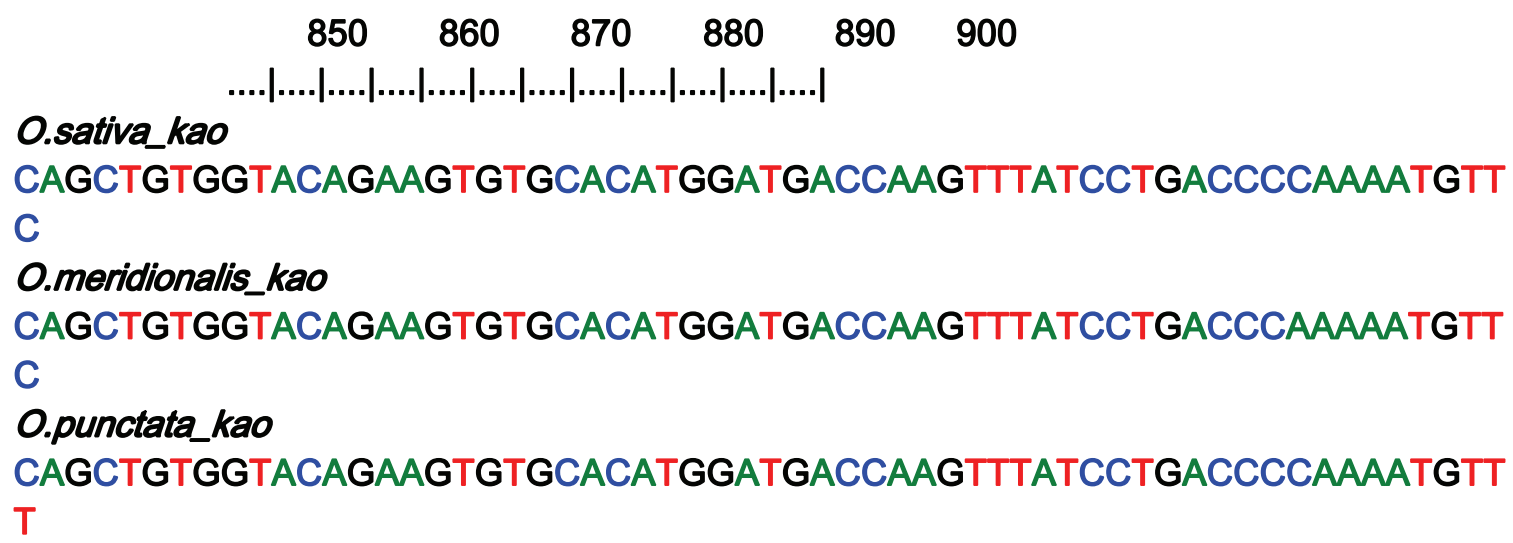

$\begin{array}{llllll}850 & 860 & 870 & 880 & 890 & 900\end{array}$

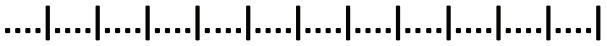

O.sativa_kao

CAGCTGTGGTACAGAAGTGTGCACATGGATGACCAAGTTTATCCTGACCCCAAAATGTT C

O.meridionalis_kao

CAGCTGTGGTACAGAAGTGTGCACATGGATGACCAAGTTTATCCTGACCCAAAAATGTT C

O.punctata_kao

CAGCTGTGGTACAGAAGTGTGCACATGGATGACCAAGTTTATCCTGACCCCAAAATGTT $\mathrm{T}$ 


\section{O.officinalis_kao}

CAGCTGTGGTACAGAAGTGTGCACATGGATGACCAAGTTTATCCTGACCCCAAAATGTT

C

O.australiensis_kao

CAGCTGTGGTATAGAAGTGTTCACATGGATGACCAAGTTTATCCTGACCCCAAAATGTT

C

O.brachyantha_kao

CAGCTGTGGTACAGAAGTGTACACATGGATGACCAAGTTTATCCTGACCCCAAAATGTT

C

O.granulata_kao

CAGCTGTGGTACAGAAGCGTGCACATGGATGACCAAGTTTATCCTGACCCCAAAGTATT

C

L.tisseranttikao

CAGTTGTGGTACAGAAGTGTGCACATGGATGACCAAGTTTATCCTGACCCCAAAACGTT

C

C.aquatica_kao

CAGCTGTGGTACAGAAGTGTGCACATGGATCCTCAAGTTTACCCTGACCCCAACAAGTT

C

R.subulata_kao

CAGCTGTGGTACAGAAGCGTGCACATGGATTCTCAAGTTTACCCTGATCCCAAAAAGTT $\mathrm{C}$

L.leiocarpa_kao

CAGCTGTGGTACAGAAGTGTGCACATGGATCCTCAAGTTTATCCTGACCCCTACAAGTT C

E.erecta_kao

CAGCTGTGGTTCAGAAATGTGCATATGGATCCTCAGGTTTATTCAGATCCCAGCAAGTT C

Clustal Consensus

O.sativa_kao

$\begin{array}{llllll}910 & 920 & 930 & 940 & 950 & 960\end{array}$ ....................................................|

AACCCTTCAAGATGGGAGGGACCCCCTCCGAAAGCCGGAACATTCCTTCCATTTGGAC TG

O.meridionalis_kao

AACCCTTCAAGATGGGAGGGACCCCCTCCGAAAGCCGGAACATTCCTTCCATTTGGAC TG

O.punctata_kao

AACCCTTCAAGATGGGAGGGCCCCCCTCCGAAAGCCGGAACATTCCTTCCATTTGGAC TG

O.officinalis_kao

AACCCTTCAAGATGGGAGGGGCCCCCTCCGAAAGCCGGAACATTCCTTCCATTTGGAC TG

O.australiensis_kao

AACCCTTCAAGATGGGAGGGTCCCCCTCCGAAAGCCGGAACATTCCTTCCATTTGGACT G 
O.brachyantha_kao

AATCCATCAAGATGGGAGGGTCCCCCGCCGAGAGCCGGAACATTCCTTCCATTTGGAC

TG

O.granulata_kao

AACCCATCAAGATGGGAGGGTCCCCCGCCGAGAGCTGGAACATTCCTTCCATTTGGAC TG

L.tisseranttikao

AACCCATCAAGATGGGAGGGTCCTCCGCCGAGAGCCGGAACATTCCTTCCATTTGGAC TG

C.aquatica_kao

AACCCTTCAAGATGGGAGGGTCCCCCGCCGAGAGCCGGAACATTCCTTCCATTTGGAC TG

R.subulata_kao

AACCCTTCAAGATGGGAGGGTCCCCCGCCGAGAGCCGGAACATTCCTTCCATTTGGAC TG

L.leiocarpa_kao

AACCCTTCAAGATGGGAGGGTCCCCCGCCAAGAGCCGGAACATTCCTCCCATTTGGAC TG

E.erecta_kao

AAGCCTTCAAGATGGGATGGTTCTTCACCGAGAGCCGGAACGTTCCTTCCATTCGGACT G

Clustal Consensus

O.sativa_kao

$\begin{array}{llllll}970 & 980 & 990 & 1000 & 1010 & 1020\end{array}$

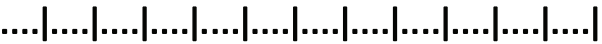

GGAGCAAGACTGTGCCCTGGAAATGATCTTGCAAAGCTGGAGATCTCTGTCTTCCTCCA $\mathrm{T}$

O.meridionalis_kao

GGAGCAAGACTGTGCCCTGGAAATGATCTTGCAAAGCTGGAGATCTCTGTCTTCCTCCA T

O.punctata_kao

GGATCGAGACTGTGCCCTGGAAATGATCTTGCAAAGCTGGAGATCTCTGTCTTCCTCCA $T$

O.officinalis_kao

GGAGCAAGACTGTGCCCTGGAAATGATCTTGCAAAGCTGGAGATCTCTGTCTTCCTCCA $\mathrm{T}$

O.australiensis_kao

GGAGCGAGGCTGTGCCCTGGAAATGATCTTGCAAAGCTGGAGATCTCTGTCTTCCTCC AT

O.brachyantha_kao GGAGCGAGACTGTGCCCTGGAAATGATCTTGCAAAGCTGGAGATCTCTGTCTTTCTCCA $\mathrm{T}$

O.granulata_kao

GGAGCGAGACTATGCCCTGGAAATGATCTTGCAAAGCTGGAGATCTCTGTCTTCCTCCA $\mathrm{T}$ 


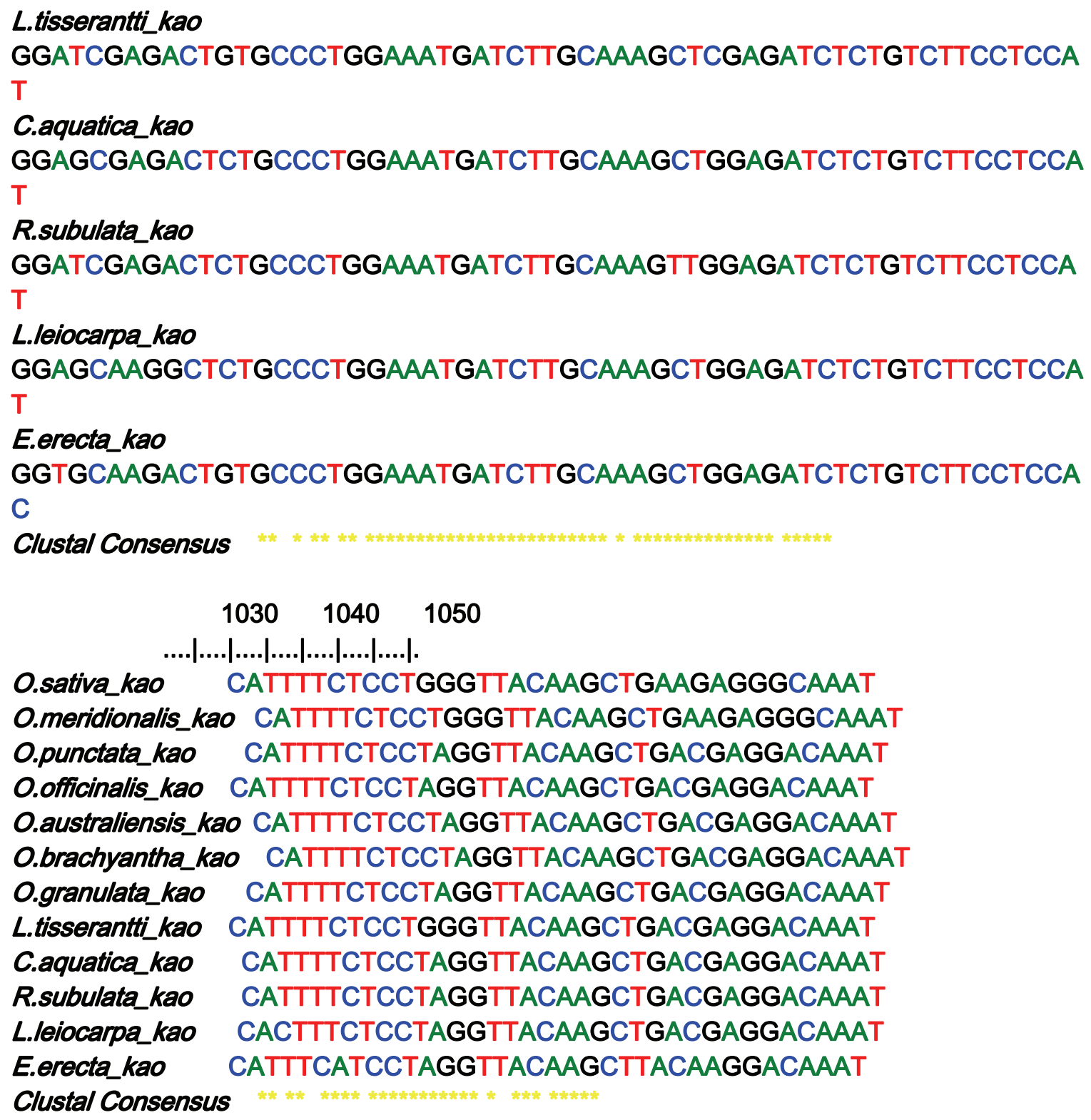

Figure S2 Alignment of coding sequences of $K A O$ gene in twelve species. Highly conserved sites are indicated with asterisks in the bottom. 